\title{
Reprogramming cell fate: a changing story
}

\section{Michael T. Chin*}

Division of Cardiology, Department of Medicine, University of Washington, Seattle, WA, USA

\section{Edited by:}

Chin-Hsing Annie Lin, University of

Texas, USA

Reviewed by:

Ravi Goyal, Loma Linda University, USA

Hong Wang, Temple University

School of Medicine, USA

Mark Feinberg, Brigham and

Women's Hospital, USA

*Correspondence:

Michael T. Chin, Center for Cardiovascular Biology, UW

Medicine at South Lake Union, Box 358050, 850 Republican Street,

Brotman 353, Seattle, WA

98040, USA

e-mail:mtchin@u.washington.edu
Direct reprogramming of adult, lineage-determined cells from one cell fate to another has long been an elusive goal in developmental biology. Recent studies have demonstrated that forced expression of lineage-specific transcription factors in various differentiated cell types can promote the adoption of different lineages. These seminal findings have the potential to revolutionize the field of regenerative medicine by providing replacement cells for various degenerative disorders. Current reprogramming protocols, however, are inefficient in that relatively few cells in a given population can be made to undergo reprogramming and the completeness and extent of reprogramming that occurs has been questioned. At present, the fundamental molecular mechanisms involved are still being elucidated. Although the potential clinical applications are extensive, these issues will need to be addressed before direct reprogramming may be used clinically. This review will give an overview of pioneering studies in the field, will describe what is known about direct reprogramming to specific lineage types, will summarize what is known about the molecular mechanisms involved in reprogramming and will discuss challenges for the future.

\section{Keywords: direct reprogramming, transdifferentiation, lineage determination, regenerative medicine, cell fate}

\section{INTRODUCTION}

A fundamental question in cell biology is whether the acquisition of a particular cell fate during embryonic development is reversible or changeable, and to what extent. From a practical standpoint, this question is also directly relevant to regenerative biology and its potential application to clinical medicine. For many years, the answer to this question has been a qualified affirmative, although progress has been mostly limited until the last decade. The first demonstration that somatic cell nuclei could be reprogrammed to direct enucleated oocytes to form mature fertile animals was achieved in amphibians (Gurdon et al., 1958). This technology was later used to clone mammals, nearly four decades later (Campbell et al., 1996; Wakayama et al., 1998). Although these studies demonstrated the feasibility of somatic nuclear reprogramming, the overall efficiency was low $(1-2 \%)$ and worked better with nuclei from cells that were less differentiated, suggesting that epigenetic modifications are likely involved.

At the cellular level, early studies showing that 5-azacytidine treatment, which inhibits DNA methylation, could convert cultured fibroblast cell lines to myocytes, chondrocytes, and adipocytes suggested that differentiated cells could undergo transdifferentiation and that this process was under epigenetic control (Taylor and Jones, 1979). Subsequent studies on human amniocyte- mouse myocyte heterokaryons were able to demonstrate that the muscle phenotype was dominant and that cytoplasmic factors caused activation of muscle genes in the human nuclei (Blau et al., 1983). A single dominant acting bHLH transcription factor, $\mathrm{MyoD}$, was later identified by its ability to transform cultured fibroblasts into myoblasts by activating muscle-specific genes (Lassar et al., 1986; Davis et al., 1987). In other terminally differentiated cell types, MyoD could activate muscle specific genes but could not suppress the starting cell phenotype, demonstrating that there are intrinsic cellular roadblocks to reprogramming (Weintraub et al., 1989). Nevertheless, this discovery prompted searches for other dominant acting transcription factors that could single handedly transform cells from one lineage to another, however, the results were largely disappointing. In general, cell fate switching seemed to occur more readily between related cell types, presumably due to similar epigenetic landscapes. Examples include conversion of primary $\mathrm{B}$ cells to macrophages by the transcription factor C/EBPa (Xie et al., 2004), activation of erythroid-megakaryocyte gene expression in monocytes by the transcription factor GATA1 (Visvader et al., 1992; Kulessa et al., 1995; Heyworth et al., 2002) and induction of myeloid gene expression in hematopoietic precursors by the transcription factor PU.1 (Nerlov and Graf, 1998).

\section{REPROGRAMMING TO PLURIPOTENCY BY MULTIPLE TRANSCRIPTION FACTORS}

The advent of technologies that facilitated global transcriptional profiling in cells and tissues allowed researchers to identify large numbers of genes that are differentially expressed in different cell types. Presumably, some of the factors that were differentially expressed in different cell lineages would contribute to the maintenance of the particular cell type. This presumption led to a pioneering study in which 24 candidate transcription factors identified in embryonic stem cells were expressed simultaneously in fibroblasts to determine whether they could confer a pluripotent phenotype, and were then gradually reduced in number to the minimum necessary to induce pluripotency, resulting in the breakthrough discovery of iPS cells. In this landmark study, 
fibroblasts could be reprogrammed for the first time into pluripotent cells through the forced expression of four defined factors: Oct3/4, Sox2, Klf4, and c-Myc (Takahashi and Yamanaka, 2006). These cells could be injected into blastocysts and contribute to all three germ layers of the developing organism, and thus can be used to generate a variety of cell types for tissue regeneration. The generation of iPS cells and their potential for use in research and therapy has discussed in several recent review articles and will not be discussed in detail (Hanna et al., 2010; Robinton and Daley, 2012). iPS cells and embryonic stem cells can be differentiated directly to a variety of cell types through a process known as "directed differentiation" using defined factors such as bone morphogenetic proteins (BMPs), Activin, Wnts, and Fibroblast Growth Factors (FGFs). Although the generation of iPS cells represents a major advancement in stem cell biology, the process is inefficient and time consuming, which will be compounded if the derived iPS cells will then be used for directed differentiation. These factors can limit their practical use in clinical settings.

\section{DIRECT REPROGRAMMING OF CELL FATE FROM ONE TYPE TO ANOTHER}

Direct reprogramming will theoretically facilitate the generation of clinically relevant cell types for organ repair from abundant, easy to obtain patient-derived cells such as fibroblasts, without the need for obtaining pluripotent stem cells. Generally this is accomplished through forced expression of lineage-specific transcription factors and has been used to promote reprogramming to a variety of cell types, such as skeletal muscle (Lassar et al., 1986; Davis et al., 1987; Weintraub et al., 1989), hepatocytes (Huang et al., 2011; Sekiya and Suzuki, 2011), neurons (Vierbuchen et al., 2010), pancreatic islet cells (Ferber et al., 2000; Zhou et al., 2008), endothelial cells (Ginsberg et al., 2012), smooth muscle cells (Cordes et al., 2009; Karamariti et al., 2013), and cardiac muscle (reviewed in Addis and Epstein, 2013). Direct reprogramming is conceptually attractive because in general it does not require reversion to a pluripotent state and represents a direct conversion from one cell lineage to another. It also provides the opportunity to directly convert cells in situ, which would be important in regenerative strategies. Several excellent reviews have been published recently on this subject (Vierbuchen and Wernig, 2012; Addis and Epstein, 2013; Morris and Daley, 2013). In general, reprogramming seems to work better when the starting cells share similar embryonic germ cell layer origins, but has been demonstrated to convert fibroblasts (mesoderm) to neurons (ectoderm), indicating that conversion across germ cell layers is possible (Vierbuchen et al., 2010). Although several different types of cells can undergo direct reprogramming to many different cell types (reviewed in Morris and Daley, 2013), we will focus primarily on what is known about direct reprogramming of fibroblasts, since they are generally ubiquitous, abundant and readily available for clinical use. Reports of direct fibroblast reprogramming are summarized in Table 1. We will also focus on directing cell fate conversion to neurons and cardiac myocytes, two cell types from organs that do not regenerate well, and are thus highly relevant to clinical regenerative medicine.
Table 1 | Reports of direct reprogramming of fibroblasts.

\begin{tabular}{ll}
\hline $\begin{array}{l}\text { Reprogrammed } \\
\text { cell type }\end{array}$ & References \\
\hline Skeletal muscle & $\begin{array}{l}\text { Lassar et al., 1986; Davis et al., 1987; Weintraub } \\
\text { et al., } 1989\end{array}$ \\
Hepatocytes & Huang et al., 2011; Sekiya and Suzuki, 2011 \\
Neurons & Vierbuchen et al., 2010; Ambasudhan et al., 2011; \\
& Caiazzo et al., 2011; Pang et al., 2011; Qiang et al., \\
& 2011; Son et al., 2011; Yoo et al., 2011; Lujan et al., \\
& 2012; Liu et al., 2013 \\
& leda et al., 2010; Efe et al., 2011; Pfisterer et al., \\
& 2011; Chen et al., 2012; Inagawa et al., 2012; Islas \\
Cardiomyocytes & et al., 2012; Jayawardena et al., 2012; Protze et al., \\
& 2012; Qian et al., 2012; Song et al., 2012; Addis \\
& et al., 2013; Christoforou et al., 2013; Fu et al., \\
& 2013; Hirai et al., 2013; Nam et al., 2013; Wada \\
& et al., 2013; Hirai and Kikyo, 2014; Ifkovits et al., \\
& 2014; Muraoka et al., 2014 \\
& Cordes et al., 2009; Karamariti et al., 2013 \\
& Feng et al., 2008 \\
& Lumelsky, 2014 \\
Mitchell et al., 2014b; Zhu et al., 2014
\end{tabular}

\section{DIRECT REPROGRAMMING TO NEURONS}

Direct reprogramming of fibroblasts to neuron-like cells was first achieved by overexpression of a pool of 19 virally expressed candidate genes that were known to be neuron-specific, play a role in neuronal differentiation or implicated in epigenetic reprogramming (Vierbuchen et al., 2010). By systematic removal of specific candidate genes and repeated transduction, these investigators were further able to demonstrate that a minimal combination of three transcription factors, Ascl1, Brn2, and Myt1l were able to rapidly reprogram embryonic and neonatal mouse fibroblasts to neuron-like cells that expressed multiple neuron-specific proteins, demonstrated spontaneous action potentials and were able to form functional synapses. The majority appeared to be cortical, glutamatergic excitatory neurons. Subsequent studies were able to demonstrate that the combination of Ascl1, Lmxla, and Nurr1 can convert mouse fibroblasts to dopaminergic neurons (Caiazzo et al., 2011), the combination of Ascl1, Brn2, Myt1l, Lhx2, Hb9, Isl1, and Ngn2 can convert mouse fibroblasts to motor neurons (Son et al., 2011) and that the combination of Brn2, Sox2, and Foxg2 could convert mouse fibroblasts to neuronal precursor cells (Lujan et al., 2012). Ascl1, Brn2, and Myt1l have also been shown to directly convert striatal astrocytes into neurons in vivo (Torper et al., 2013). NeuroD has also been shown to directly reprogram reactive glial cells into functional neurons within the cerebral cortex after brain injury (Guo et al., 2014).

Parallel studies on human fibroblasts were able to show that various combinations of factors such as Ascl1, Brn2, Myt1l, and NeuroD1 (Pang et al., 2011); Ascl1, Myt1l, NeuroD2, miR-9/9, and miR-124 (Yoo et al., 2011); or Brn2, Myt1l, and miR-124 (Ambasudhan et al., 2011) could reprogram these cells to glutamatergic neurons. A group of five factors (Ascl1, Brn2, Myt1l, Olig2, and Zic1) could also reprogram human skin fibroblasts into glutamatergic neurons and was used to generate induced 
neurons from patients with Alzheimer's Disease (Qiang et al,, 2011). Similarly, the combination of Ascl1, Brn2, Myt1l, Lmxla, and Foxa2 (Pfisterer et al., 2011) or the combination of Ascll, Lmxla, and Nurr1 (Caiazzo et al., 2011) could promote the formation of dopaminergic neurons from human fibroblasts. Human fibroblasts could also be directly reprogrammed into motor neurons by the combination of Ascl1, Brn2, Myt1l, Lhx2, Hb9, Isl1, and Ngn2 (Son et al., 2011).

\section{DIRECT REPROGRAMMING OF FIBROBLASTS TO CARDIOMYOCYTES}

The first demonstration that mouse fibroblasts could be directly reprogrammed to induced cardiac myocyte-like cells (iCMs) was achieved using an approach similar to that used to generate iPS cells and induced neuronal cells. A pool of 14 candidate factors was initially shown to induce cardiomyocyte-like cells and then the pool was narrowed down to the combination of Gata4, Mef2c, and Tbx5 (GMT) (Ieda et al., 2010). Only a small percentage of fibroblasts were directly reprogrammed, however, and although they had many features of cardiac myocytes, their transcriptional patterns were distinct from neonatal cardiomyocytes. In addition, only a small percentage of the cells could spontaneously contract. Another approach using a different strategy of transiently expressing the pluripotency factors Oct4, Sox2, Klf4, and c-Myc, then culturing the cells in defined media conditions commonly used in the stem cell field to promote cardiac differentiation, including the JAK inhibitor JI1, was also successful (Efe et al., 2011). Another group reported that the GMT factor combination was able to induce expression of cardiac genes, but did not produce any contracting cells (Chen et al., 2012), raising doubts about the efficacy and efficiency of the procedure. Two subsequent studies, however, were able to demonstrate that the retroviral expression of GMT transcription factors could directly reprogram fibroblasts at the site of myocardial injury and decrease infarct size, especially when given in conjunction with thymosin $\beta 4$ (Inagawa et al., 2012; Qian et al., 2012). A different group reported that direct reprogramming of mouse fibroblasts was more efficient if the transcription factor Hand2 was added in conjunction with GMT, both in vitro and in vivo after myocardial injury (Song et al., 2012). A subsequent study evaluated the effect of three factor combinations from a pool of 10 candidate factors and determined that Tbx 5 , Mef2c, and Myocardin induced a broader spectrum of myocardial genes than Gata4, Mef2c, and Tbx5 (Protze et al., 2012). Another study investigated the potential for microRNAs to reprogram mouse fibroblasts to cardiac myocyte like cells and determined that the combination of miR-1, miR-133, miR-208, and miR-499, in conjunction with JAK inhibitor I was sufficient both in vitro and in vivo (Jayawardena et al., 2012). Others have tried to optimize the reprogramming further and have found that addition of Myocardin, SRF, Mesp1, and Smarcd2 to Gata4, Mef2c, and Tbx5 can enhance the process (Christoforou et al., 2013). To improve the likelihood of obtaining functional cardiac myocytes, another group used fibroblasts containing a calcium sensitive GFP reporter and found that the combination of Hand2, Nkx2-5, Gata4, Mef2c, and Tbx 5 could reprogram adult mouse fibroblasts 50 fold more efficiently than GMT alone and that the induced cardiac myocytes demonstrated robust calcium oscillations and spontaneous beating (Addis et al., 2013). The efficiency of conversion by GMT to spontaneously contracting cardiomyocyte-like cells was also reportedly improved by the tethering of the MyoD activation domain to each of these transcription factors (Hirai et al., 2013). A follow up study showed that direct reprogramming with these factors was further enhanced by inhibition of repressive histone modifications (Hirai and Kikyo, 2014).

Direct reprogramming of human fibroblasts to cardiac myocyte-like cells has also been reported, but with different factor requirements. Forced expression of the transcription factors Ets2 and Mesp1 or recombinant ETS2 and MESP1 proteins modified with cell penetrating peptides were sufficient to convert human neonatal foreskin fibroblasts into cardiac progenitors (Islas et al., 2012). The transcription factors Gata4, Hand2, myocardin, and Tbx5 in conjunction with microRNAs miR-1 and miR-133 were sufficient to directly reprogram neonatal foreskin, adult cardiac and adult dermal fibroblasts to cardiomyocyte-like cells (Nam et al., 2013). The function of miR-133 in this context is reportedly to suppress Snail and fibroblast genes (Muraoka et al., 2014). The addition of Myocardin and Mesp1 to GMT was reported to reprogram human cardiac fibroblasts to cardiomyocyte-like cells that express a broad array of cardiac genes and exhibit calcium oscillations (Wada et al., 2013). GMT factors in conjunction with MESP1 and ESRRG have also been reported to directly reprogram several types of human fibroblasts to cardiomyocyte-like cells (Fu et al., 2013).

These studies in aggregate demonstrate that multiple transcription factors and microRNAs can contribute to direct reprogramming of fibroblasts. One potential contributor to the variation between these studies is the lack of consensus criteria for assessing the degree of reprogramming. The development and use of standardized criteria for evaluation of transdifferentiation to iCMs, in terms of gene expression, structural, and functional characteristics has been suggested for these types of experiments (Addis and Epstein, 2013).

\section{MECHANISMS OF DIRECT REPROGRAMMING}

The mechanisms of direct reprogramming are incompletely understood. While it is well established that transcription factors drive the process and that microRNAs can contribute, it is less clear how cells maintain lineage and in general prevent the development of inappropriate cell types. The process involves activation of target genes, which usually occurs within hours to days (Ieda et al., 2010; Vierbuchen et al., 2010), direct transition from one state to another, without the need to go through a pluripotent state (Zhou et al., 2008; Ieda et al., 2010), does not require cell division, in contrast to induction of pluripotency (Zhou et al., 2008; Hanna et al., 2009; Heinrich et al., 2010; Vierbuchen et al., 2010) and is stable after removal of reprogramming factors (Zhou et al., 2008; Huang et al., 2011; Sekiya and Suzuki, 2011). The interactions between the positive actions of transcription factors and the negative influences of chromatin architecture and epigenetic modifications are currently under investigation. It has long been known that the genome encodes many binding sites for a given transcription factor, but the local chromatin structure only allows certain sites to be accessible, 
in a cell type-specific fashion. An example is the hematopoietic transcription factor Scl/Tal, which binds to different sites in different hematopoietic cell types (Wilson et al., 2010; Palii et al., 2011). Unneeded areas of the genome are packaged into heterochromatin and are generally not accessible to transcription factors (Beisel and Paro, 2011). To achieve reprogramming, not only must the reprogramming factors find appropriate binding sites, they must also remodel chromatin appropriately to allow ancillary factors to bind and activate a cell type-specific program. This challenge may explain the general requirement during direct reprogramming for multiple transcription factors that act cooperatively to remodel diverse areas throughout the genome. Another hypothesis being considered is that the reprogramming factors act as "pioneer" transcription factors that can bind to their cognate sites regardless of chromatin configuration (Zaret and Carroll, 2011). In this model, the pioneering factors can bind to their cognate sites and displace nucleosomes, thereby creating a permissive environment for other factors to bind. Given that some cell types are not amenable to direct reprogramming and that related cells are generally more amenable to reprogramming, it is likely that some degree of initial chromatin accessibility or "open access" is necessary even for factors that have "pioneer" capability. Studies on the muscle specific factor MyoD demonstrate that cells susceptible to reprogramming have accessible enhancer elements that allow MyoD binding despite being in an overall repressive state where gene transcription is turned off. Ectopic MyoD was able to quickly bind the enhancer element in the first $24 \mathrm{~h}$, followed by acquisition of H3K4me marks by $48 \mathrm{~h}$ (Taberlay et al., 2011).

Direct reprogramming to different cell types occurs at varying efficiency but is usually low. In addition, successful reprogramming often requires high expression levels of reprogramming factors. Accordingly, another postulated mechanism of reprogramming involves transient accessibility to transcription factor binding sites during nucleosome turnover or other mechanisms in which DNA becomes accessible in a stochastic fashion, such as during different phases of the cell cycle (Egli et al., 2008; Vierbuchen and Wernig, 2012).

\section{CURRENT LIMITATIONS AND CHALLENGES FOR THE FUTURE}

In addition to low efficiency, another major limitation of direct reprogramming as a strategy to regenerate tissues is the presence of epigenetic memory. Epigenetic memory specific to the original cell type has been well documented in iPS cells (Kim et al., 2010, 2011; Polo et al., 2010). Despite induction of gene expression consistent with reprogramming to another cell type, in multiple cases, some residual gene expression specific to the cell type of origin persists (Feng et al., 2008; Marro et al., 2011). Induced neurons derived from hepatocytes still demonstrate some hepatocyte-specific gene expression (Marro et al., 2011), while induced macrophages derived from fibroblasts still express some fibroblast genes (Feng et al., 2008). In many reported cases of direct reprogramming, only a small set of target genes were assessed, and in cases where more thorough transcriptomic analysis has been performed, there is significant divergence in gene expression patterns from native cells (Ieda et al., 2010; Sekiya and Suzuki, 2011). Since epigenetic memory has also been shown to persist in embryos generated from somatic cell nuclei ( $\mathrm{Ng}$ and Gurdon, 2005), this problem may be challenging to resolve.

A promising alternative approach has been to use pluripotency factors in the early stage of direct reprogramming followed by induction with cell-type specific factors to promote the differentiation of fibroblasts to cardiac myocytes (Efe et al., 2011). This method is thought to induce a transient state of plasticity more amenable to direct reprogramming without full induction of pluripotency, and reportedly is much more efficient than direct reprogramming. Oct4 in particular has been implicated to play an important role in this regard (Mitchell et al., 2014a,b). To date, however, this approach is limited by persistence of pluripotency markers in the reprogrammed cells and the resulting cells have properties of atrial cardiac myocytes, which may be less useful for regenerative purposes. In general, the phenotype of directly reprogrammed cells is often immature compared to fully differentiated native cells within the target organ of interest, and this may limit their utility in regenerative medicine. For cardiac cells in particular, incomplete differentiation may prevent proper electrical and mechanical coupling, leading to arrhythmias and possibly heart failure. Strategies to promote a state of differentiation comparable to that of target tissue will also be critical to facilitate the use of these cells in regenerative medicine. Exogenous, chemically defined components such as ascorbic acid, recombinant human albumin and other small molecules may be useful in this regard (Crescini et al., 2013; Burridge et al., 2014). The utility of small molecules and chemically defined conditions in promoting direct reprogramming is well established (Lin et al., 2013; Liu et al., 2013; Ifkovits et al., 2014; Lumelsky, 2014; Zhu et al., 2014).

Overall, the potential applications of direct reprogramming to regenerative medicine are extensive. More studies are needed, however, to characterize more fully the phenotype of reprogrammed cells, particularly the extent of epigenetic memory, residual gene expression specific to the original cell type and ability to achieve an appropriate differentiation state and function similarly to native cells. Further refinement of transcription factor combinations, the use of adjunct agents that promote chromatin accessibility, the use of small molecules and the potential utility of pluripotency factors are only a few of the possible approaches to enhance direct reprogramming that are expected to evolve in the future.

\section{REFERENCES}

Addis, R. C., and Epstein, J. A. (2013). Induced regeneration-the progress and promise of direct reprogramming for heart repair. Nat. Med. 19, 829-836. doi: 10.1038/nm.3225

Addis, R. C., Ifkovits, J. L., Pinto, F., Kellam, L. D., Esteso, P., Rentschler, S., et al. (2013). Optimization of direct fibroblast reprogramming to cardiomyocytes using calcium activity as a functional measure of success. J. Mol. Cell. Cardiol. 60, 97-106. doi: 10.1016/j.yjmcc.2013.04.004

Ambasudhan, R., Talantova, M., Coleman, R., Yuan, X., Zhu, S., Lipton, S. A., et al. (2011). Direct reprogramming of adult human fibroblasts to functional neurons under defined conditions. Cell Stem Cell 9, 113-118. doi: 10.1016/j.stem.2011.07.002

Beisel, C., and Paro, R. (2011). Silencing chromatin: comparing modes and mechanisms. Nat. Rev. Genet. 12, 123-135. doi: 10.1038/nrg2932

Blau, H. M., Chiu, C. P., and Webster, C. (1983). Cytoplasmic activation of human nuclear genes in stable heterocaryons. Cell 32, 1171-1180. doi: 10.1016/00928674(83)90300-8 
Burridge, P. W., Matsa, E., Shukla, P., Lin, Z. C., Churko, J. M., Ebert, A. D., et al. (2014). Chemically defined generation of human cardiomyocytes. Nat. Methods 11, 855-860. doi: 10.1038/nmeth.2999

Caiazzo, M., Dell'Anno, M. T., Dvoretskova, E., Lazarevic, D., Taverna, S., Leo, D., et al. (2011). Direct generation of functional dopaminergic neurons from mouse and human fibroblasts. Nature 476, 224-227. doi: 10.1038/nature10284

Campbell, K. H., McWhir, J., Ritchie, W. A., and Wilmut, I. (1996). Sheep cloned by nuclear transfer from a cultured cell line. Nature 380, 64-66. doi: $10.1038 / 380064 \mathrm{a} 0$

Chen, J. X., Krane, M., Deutsch, M. A., Wang, L., Rav-Acha, M., Gregoire, S., et al. (2012). Inefficient reprogramming of fibroblasts into cardiomyocytes using Gata4, Mef2c, and Tbx5. Circ. Res. 111, 50-55. doi: 10.1161/CIRCRESAHA.112.270264

Christoforou, N., Chellappan, M., Adler, A. F., Kirkton, R. D., Wu, T., Addis, R. C., et al. (2013). Transcription factors MYOCD, SRF, Mesp1 and SMARCD3 enhance the cardio-inducing effect of GATA4, TBX5, and MEF2C during direct cellular reprogramming. PLoS ONE 8:e63577. doi: 10.1371/journal.pone. 0063577

Cordes, K. R., Sheehy, N. T., White, M. P., Berry, E. C., Morton, S. U., Muth, A. N., et al. (2009). miR-145 and miR-143 regulate smooth muscle cell fate and plasticity. Nature 460, 705-710. doi: 10.1038/nature08195

Crescini, E., Gualandi, L., Uberti, D., Prandelli, C., Presta, M., and Dell'Era, P. (2013). Ascorbic acid rescues cardiomyocyte development in Fgfr1(-/-) murine embryonic stem cells. Biochim. Biophys. Acta 1833, 140-147. doi: 10.1016/j.bbamcr.2012.06.024

Davis, R. L., Weintraub, H., and Lassar, A. B. (1987). Expression of a single transfected cDNA converts fibroblasts to myoblasts. Cell 51, 987-1000. doi: 10.1016/0092-8674(87)90585-X

Efe, J. A., Hilcove, S., Kim, J., Zhou, H., Ouyang, K., Wang, G., et al. (2011). Conversion of mouse fibroblasts into cardiomyocytes using a direct reprogramming strategy. Nat. Cell Biol. 13, 215-222. doi: 10.1038/ncb2164

Egli, D., Birkhoff, G., and Eggan, K. (2008). Mediators of reprogramming: transcription factors and transitions through mitosis. Nat. Rev. Mol. Cell Biol. 9, 505-516. doi: 10.1038/nrm2439

Feng, R., Desbordes, S. C., Xie, H., Tillo, E. S., Pixley, F., Stanley, E. R., et al. (2008). PU.1 and C/EBPalpha/beta convert fibroblasts into macrophage-like cells. Proc Natl. Acad. Sci. U.S.A. 105, 6057-6062. doi: 10.1073/pnas.0711961105

Ferber, S., Halkin, A., Cohen, H., Ber, I., Einav, Y., Goldberg, I., et al. (2000). Pancreatic and duodenal homeobox gene 1 induces expression of insulin genes in liver and ameliorates streptozotocin-induced hyperglycemia. Nat. Med. 6 , 568-572. doi: 10.1038/75050

Fu, J. D., Stone, N. R., Liu, L., Spencer, C. I., Qian, L., Hayashi, Y., et al. (2013). Direct reprogramming of human fibroblasts toward a cardiomyocyte-like state. Stem Cell Reports 1, 235-247. doi: 10.1016/j.stemcr.2013.07.005

Ginsberg, M., James, D., Ding, B. S., Nolan, D., Geng, F., Butler, J. M., et al. (2012). Efficient direct reprogramming of mature amniotic cells into endothelial cells by ETS factors and TGFbeta suppression. Cell 151, 559-575. doi: 10.1016/j.cell.2012.09.032

Guo, Z., Zhang, L., Wu, Z., Chen, Y., Wang, F., and Chen, G. (2014). In vivo direct reprogramming of reactive glial cells into functional neurons after brain injury and in an Alzheimer's disease model. Cell Stem Cell 14, 188-202. doi: 10.1016/j.stem.2013.12.001

Gurdon, J. B., Elsdale, T. R., and Fischberg, M. (1958). Sexually mature individuals of Xenopus laevis from the transplantation of single somatic nuclei. Nature 182, 64-65. doi: 10.1038/182064a0

Hanna, J., Saha, K., Pando, B., van Zon, J., Lengner, C. J., Creyghton, M. P., et al. (2009). Direct cell reprogramming is a stochastic process amenable to acceleration. Nature 462, 595-601. doi: 10.1038/nature08592

Hanna, J. H., Saha, K., and Jaenisch, R. (2010). Pluripotency and cellular reprogramming: facts, hypotheses, unresolved issues. Cell 143, 508-525. doi: 10.1016/i.cell.2010.10.008

Heinrich, C., Blum, R., Gascon, S., Masserdotti, G., Tripathi, P., Sanchez, R., et al. (2010). Directing astroglia from the cerebral cortex into subtype specific functional neurons. PLoS Biol. 8:e1000373. doi: 10.1371/journal.pbio.1000373

Heyworth, C., Pearson, S., May, G., and Enver, T. (2002). Transcription factormediated lineage switching reveals plasticity in primary committed progenitor cells. EMBO J. 21, 3770-3781. doi: 10.1093/emboj/cdf368

Hirai, H., Katoku-Kikyo, N., Keirstead, S. A., and Kikyo, N. (2013). Accelerated direct reprogramming of fibroblasts into cardiomyocyte-like cells with the MyoD transactivation domain. Cardiovasc. Res. 100, 105-113. doi: $10.1093 / \mathrm{cvr} / \mathrm{cvt} 167$

Hirai, H., and Kikyo, N. (2014). Inhibitors of suppressive histone modification promote direct reprogramming of fibroblasts to cardiomyocyte-like cells. Cardiovasc. Res. 102, 188-190. doi: 10.1093/cvr/cvu023

Huang, P., He, Z., Ji, S., Sun, H., Xiang, D., Liu, C., et al. (2011). Induction of functional hepatocyte-like cells from mouse fibroblasts by defined factors. Nature 475, 386-389. doi: 10.1038/nature 10116

Ieda, M., Fu, J. D., Delgado-Olguin, P., Vedantham, V., Hayashi, Y., Bruneau, B. G., et al. (2010). Direct reprogramming of fibroblasts into functional cardiomyocytes by defined factors. Cell 142, 375-386. doi: 10.1016/j.cell.2010.07.002

Ifkovits, J. L., Addis, R. C., Epstein, J. A., and Gearhart, J. D. (2014). Inhibition of TGFbeta signaling increases direct conversion of fibroblasts to induced cardiomyocytes. PLoS ONE 9:e89678. doi: 10.1371/journal.pone.0089678

Inagawa, K., Miyamoto, K., Yamakawa, H., Muraoka, N., Sadahiro, T., Umei, T., et al. (2012). Induction of cardiomyocyte-like cells in infarct hearts by gene transfer of Gata4, Mef2c, and Tbx5. Circ. Res. 111, 1147-1156. doi: 10.1161/CIRCRESAHA.112.271148

Islas, J. F., Liu, Y., Weng, K. C., Robertson, M. J., Zhang, S., Prejusa, A., et al. (2012). Transcription factors ETS2 and MESP1 transdifferentiate human dermal fibroblasts into cardiac progenitors. Proc. Natl. Acad. Sci. U.S.A. 109, 13016-13021. doi: $10.1073 /$ pnas.1120299109

Jayawardena, T. M., Egemnazarov, B., Finch, E. A., Zhang, L., Payne, J. A., Pandya, K., et al. (2012). MicroRNA-mediated in vitro and in vivo direct reprogramming of cardiac fibroblasts to cardiomyocytes. Circ. Res. 110, 1465-1473. doi: 10.1161/CIRCRESAHA.112.269035

Karamariti, E., Margariti, A., Winkler, B., Wang, X., Hong, X., Baban, D., et al. (2013). Smooth muscle cells differentiated from reprogrammed embryonic lung fibroblasts through DKK3 signaling are potent for tissue engineering of vascular grafts. Circ. Res. 112, 1433-1443. doi: 10.1161/CIRCRESAHA.111.300415

Kim, K., Doi, A., Wen, B., Ng, K., Zhao, R., Cahan, P., et al. (2010). Epigenetic memory in induced pluripotent stem cells. Nature 467, 285-290. doi: 10.1038/nature09342

Kim, K., Zhao, R., Doi, A., Ng, K., Unternaehrer, J., Cahan, P., et al. (2011). Donor cell type can influence the epigenome and differentiation potential of human induced pluripotent stem cells. Nat. Biotechnol. 29, 1117-1119. doi: $10.1038 /$ nbt.2052

Kulessa, H., Frampton, J., and Graf, T. (1995). GATA-1 reprograms avian myelomonocytic cell lines into eosinophils, thromboblasts, and erythroblasts. Genes Dev. 9, 1250-1262. doi: 10.1101/gad.9.10.1250

Lassar, A. B., Paterson, B. M., and Weintraub, H. (1986). Transfection of a DNA locus that mediates the conversion of $10 \mathrm{~T} 1 / 2$ fibroblasts to myoblasts. Cell 47 , 649-656. doi: 10.1016/0092-8674(86)90507-6

Lin, C., Yu, C., and Ding, S. (2013). Toward directed reprogramming through exogenous factors. Curr. Opin. Genet. Dev. 23, 519-525. doi: 10.1016/j.gde.2013.06.002

Liu, M. L., Zang, T., Zou, Y., Chang, J. C., Gibson, J. R., Huber, K. M., et al. (2013). Small molecules enable neurogenin 2 to efficiently convert human fibroblasts into cholinergic neurons. Nat. Commun. 4, 2183. doi: 10.1038/ncomms3183

Lujan, E., Chanda, S., Ahlenius, H., Sudhof, T. C., and Wernig, M. (2012). Direct conversion of mouse fibroblasts to self-renewing, tripotent neural precursor cells. Proc. Natl. Acad. Sci. U.S.A. 109, 2527-2532. doi: 10.1073/pnas.1121003109

Lumelsky, N. (2014). Small molecules convert fibroblasts into islet-like cells avoiding pluripotent state. Cell Metab. 19, 551-552. doi: 10.1016/j.cmet.2014.03.019

Marro, S., Pang, Z. P., Yang, N., Tsai, M. C., Qu, K., Chang, H. Y., et al. (2011). Direct lineage conversion of terminally differentiated hepatocytes to functional neurons. Cell Stem Cell 9, 374-382. doi: 10.1016/j.stem.2011.09.002

Mitchell, R., Szabo, E., Shapovalova, Z., Aslostovar, L., Makondo, K., and Bhatia, M. (2014a). Molecular evidence for OCT4 induced plasticity in adult human fibroblasts required for direct cell fate conversion to lineage specific progenitors. Stem Cells 32, 2178-2187. doi: 10.1002/stem.1721

Mitchell, R. R., Szabo, E., Benoit, Y. D., Case, D. T., Mechael, R., Alamilla, J., et al. (2014b). Activation of neural cell fate programs toward direct conversion of adult human fibroblasts into Tri-potent neural progenitors using OCT-4. Stem Cells Dev. 23, 1937-1946. doi: 10.1089/scd.2014.0023

Morris, S. A., and Daley, G. Q. (2013). A blueprint for engineering cell fate: current technologies to reprogram cell identity. Cell Res. 23, 33-48. doi: $10.1038 / \mathrm{cr} .2013 .1$ 
Muraoka, N., Yamakawa, H., Miyamoto, K., Sadahiro, T., Umei, T., Isomi, M., et al. (2014). MiR-133 promotes cardiac reprogramming by directly repressing Snail and silencing fibroblast signatures. EMBO J. 33, 1565-1581. doi: 10.15252/embj.201387605

Nam, Y. J., Song, K., Luo, X., Daniel, E., Lambeth, K., West, K., et al. (2013). Reprogramming of human fibroblasts toward a cardiac fate. Proc. Natl. Acad. Sci. U.S.A. 110, 5588-5593. doi: 10.1073/pnas.1301019110

Nerlov, C., and Graf, T. (1998). PU.1 induces myeloid lineage commitment in multipotent hematopoietic progenitors. Genes Dev. 12, 2403-2412. doi: 10.1101/gad.12.15.2403

Ng, R. K., and Gurdon, J. B. (2005). Epigenetic memory of active gene transcription is inherited through somatic cell nuclear transfer. Proc. Natl. Acad. Sci. U.S.A. 102, 1957-1962. doi: 10.1073/pnas.0409813102

Palii, C. G., Perez-Iratxeta, C., Yao, Z., Cao, Y., Dai, F., Davison, J., et al. (2011). Differential genomic targeting of the transcription factor TAL1 in alternate haematopoietic lineages. EMBO J. 30, 494-509. doi: 10.1038/emboj.2010.342

Pang, Z. P., Yang, N., Vierbuchen, T., Ostermeier, A., Fuentes, D. R., Yang, T. Q., et al. (2011). Induction of human neuronal cells by defined transcription factors. Nature 476, 220-223. doi: 10.1038/nature10202

Pfisterer, U., Kirkeby, A., Torper, O., Wood, J., Nelander, J., Dufour, A., et al. (2011) Direct conversion of human fibroblasts to dopaminergic neurons. Proc. Natl. Acad. Sci. U.S.A. 108, 10343-10348. doi: 10.1073/pnas.1105135108

Polo, J. M., Liu, S., Figueroa, M. E., Kulalert, W., Eminli, S., Tan, K. Y., et al. (2010). Cell type of origin influences the molecular and functional properties of mouse induced pluripotent stem cells. Nat. Biotechnol. 28, 848-855. doi: $10.1038 /$ nbt. 1667

Protze, S., Khattak, S., Poulet, C., Lindemann, D., Tanaka, E. M., and Ravens, U. (2012). A new approach to transcription factor screening for reprogramming of fibroblasts to cardiomyocyte-like cells. J. Mol. Cell. Cardiol. 53, 323-332. doi: 10.1016/j.yjmcc.2012.04.010

Qian, L., Huang, Y., Spencer, C. I., Foley, A., Vedantham, V., Liu, L., et al. (2012). In vivo reprogramming of murine cardiac fibroblasts into induced cardiomyocytes. Nature 485, 593-598. doi: 10.1038/nature11044

Qiang, L., Fujita, R., Yamashita, T., Angulo, S., Rhinn, H., Rhee, D., et al. (2011). Directed conversion of Alzheimer's disease patient skin fibroblasts into functional neurons. Cell 146, 359-371. doi: 10.1016/j.cell.2011.07.007

Robinton, D. A., and Daley, G. Q. (2012). The promise of induced pluripotent stem cells in research and therapy. Nature 481, 295-305. doi: 10.1038/nature10761

Sekiya, S., and Suzuki, A. (2011). Direct conversion of mouse fibroblasts to hepatocyte-like cells by defined factors. Nature 475, 390-393. doi: 10.1038/nature10263

Son, E. Y., Ichida, J. K., Wainger, B. J., Toma, J. S., Rafuse, V. F., Woolf, C. J., et al. (2011). Conversion of mouse and human fibroblasts into functional spinal motor neurons. Cell Stem Cell 9, 205-218. doi: 10.1016/j.stem.2011.07.014

Song, K., Nam, Y. J., Luo, X., Qi, X., Tan, W., Huang, G. N., et al. (2012). Heart repair by reprogramming non-myocytes with cardiac transcription factors. Nature 485, 599-604. doi: 10.1038/nature11139

Taberlay, P. C., Kelly, T. K., Liu, C. C., You, J. S., De Carvalho, D. D., Miranda, T. B., et al. (2011). Polycomb-repressed genes have permissive enhancers that initiate reprogramming. Cell 147, 1283-1294. doi: 10.1016/j.cell.2011.10.040

Takahashi, K., and Yamanaka, S. (2006). Induction of pluripotent stem cells from mouse embryonic and adult fibroblast cultures by defined factors. Cell 126 , 663-676. doi: 10.1016/j.cell.2006.07.024

Taylor, S. M., and Jones, P. A. (1979). Multiple new phenotypes induced in 10T1/2 and 3 T3 cells treated with 5-azacytidine. Cell 17, 771-779. doi: 10.1016/00928674(79)90317-9
Torper, O., Pfisterer, U., Wolf, D. A., Pereira, M., Lau, S., Jakobsson, J., et al. (2013). Generation of induced neurons via direct conversion in vivo. Proc. Natl. Acad. Sci. U.S.A. 110, 7038-7043. doi: 10.1073/pnas.1303829110

Vierbuchen, T., Ostermeier, A., Pang, Z. P., Kokubu, Y., Sudhof, T. C., and Wernig, M. (2010). Direct conversion of fibroblasts to functional neurons by defined factors. Nature 463, 1035-1041. doi: 10.1038/nature08797

Vierbuchen, T., and Wernig, M. (2012). Molecular roadblocks for cellular reprogramming. Mol. Cell 47, 827-838. doi: 10.1016/j.molcel.2012.09.008

Visvader, J. E., Elefanty, A. G., Strasser, A., and Adams, J. M. (1992). GATA-1 but not SCL induces megakaryocytic differentiation in an early myeloid line. $E M B O$ J. 11, 4557-4564.

Wada, R., Muraoka, N., Inagawa, K., Yamakawa, H., Miyamoto, K., Sadahiro, T., et al. (2013). Induction of human cardiomyocyte-like cells from fibroblasts by defined factors. Proc. Natl. Acad. Sci. U.S.A. 110, 12667-12672. doi: 10.1073/pnas.1304053110

Wakayama, T., Perry, A. C., Zuccotti, M., Johnson, K. R., and Yanagimachi, R. (1998). Full-term development of mice from enucleated oocytes injected with cumulus cell nuclei. Nature 394, 369-374. doi: 10.1038/28615

Weintraub, H., Tapscott, S. J., Davis, R. L., Thayer, M. J., Adam, M. A., Lassar, A. B., et al. (1989). Activation of muscle-specific genes in pigment, nerve, fat, liver, and fibroblast cell lines by forced expression of MyoD. Proc. Natl. Acad. Sci. U.S.A 86, 5434-5438. doi: 10.1073/pnas.86.14.5434

Wilson, N. K., Foster, S. D., Wang, X., Knezevic, K., Schutte, J., Kaimakis, P., et al. (2010). Combinatorial transcriptional control in blood stem/progenitor cells: genome-wide analysis of ten major transcriptional regulators. Cell Stem Cell 7, 532-544. doi: 10.1016/j.stem.2010.07.016

Xie, H., Ye, M., Feng, R., and Graf, T. (2004). Stepwise reprogramming of B cells into macrophages. Cell 117, 663-676. doi: 10.1016/S0092-8674(04)00419-2

Yoo, A. S., Sun, A. X., Li, L., Shcheglovitov, A., Portmann, T., Li, Y., et al. (2011). MicroRNA-mediated conversion of human fibroblasts to neurons. Nature 476, 228-231. doi: 10.1038/nature10323

Zaret, K. S., and Carroll, J. S. (2011). Pioneer transcription factors: establishing competence for gene expression. Genes Dev. 25, 2227-2241. doi: 10.1101/gad.176826.111

Zhou, Q., Brown, J., Kanarek, A., Rajagopal, J., and Melton, D. A. (2008). In vivo reprogramming of adult pancreatic exocrine cells to beta-cells. Nature 455, 627-632. doi: 10.1038/nature07314

Zhu, S., Ambasudhan, R., Sun, W., Kim, H. J., Talantova, M., Wang, X., et al. (2014) Small molecules enable OCT4-mediated direct reprogramming into expandable human neural stem cells. Cell Res. 24, 126-129. doi: 10.1038/cr.2013.156

Conflict of Interest Statement: The author declares that the research was conducted in the absence of any commercial or financial relationships that could be construed as a potential conflict of interest.

Received: 21 July 2014; accepted: 11 August 2014; published online: 27 August 2014. Citation: Chin MT (2014) Reprogramming cell fate: a changing story. Front. Cell Dev. Biol. 2:46. doi: 10.3389/fcell.2014.00046

This article was submitted to Epigenomics and Epigenetics, a section of the journal Frontiers in Cell and Developmental Biology.

Copyright (c) 2014 Chin. This is an open-access article distributed under the terms of the Creative Commons Attribution License (CC BY). The use, distribution or reproduction in other forums is permitted, provided the original author(s) or licensor are credited and that the original publication in this journal is cited, in accordance with accepted academic practice. No use, distribution or reproduction is permitted which does not comply with these terms. 